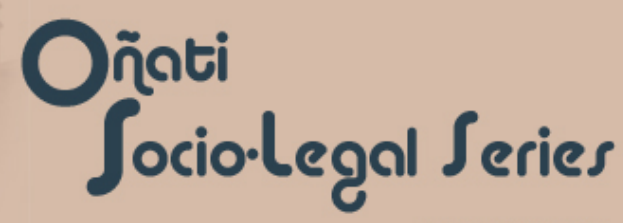

ISSN: 2079-5971

Oñati Socio-legal Series, v. 9, n. 4 (2019) - La institucionalización de la mediación: potencialidades y riesgos

ISSN: 2079-5971

\title{
Démocratie, institution, marché. Les vents contraires de la médiation (Democracy, institution, market: the conflicting winds of mediation)
}

JACQUES FAGET*

Faget, J., 2019. Démocratie, institution, marché. Les vents contraires de la médiation Received 21 February 2018, Accepted 10 January 2019. Oñati Socio-legal Series [online], 9(4), 429-444. Available from: https://doi.org/10.35295/osls.iisl/0000-0000-0000-1079

\section{Résumé}

Le développement considérable des médiations depuis quelques décennies dans les pays occidentaux est corrélé avec les insuffisances et l'anachronisme de régulations institutionnelles et sociales bousculées par la postmodernité. Les métamorphoses engendrées par leur succès ne se font pas sans heurts entre des juristes fragilisés par la crise de la rationalité juridique défendant leur monopole et les nouveaux professionnels de la médiation venant des sciences humaines. C'est ainsi que s'affrontent deux conceptions de la médiation, une vision idéaliste axée sur l'empowerment et l'émancipation des individus et une vision pragmatique ou marchande qui tend à juridiciser toujours plus les modes alternatifs de résolution des conflits.

\section{Mots clés}

Médiation; institutionnalisation; postmodernité; néolibéralisme; empowerment; démocratie; marché

\section{Abstract}

The considerable development of mediations over the past few decades in Western countries is correlated with the insufficiencies and anachronism of institutional and social regulations knocked over by postmodernity. The metamorphoses generated by their success are not without struggle between jurists weakened by the crisis of legal rationality, defending their monopoly, and the new professionals of mediation coming from social sciences. Thus, two conceptions of mediation confront one another, an idealistic vision of empowerment and emancipation of individuals, and a pragmatic or marketably vision that tends to juridicize alternatives dispute resolution.

\section{Key words}

Mediation; institutionalization; post-modernity; neoliberalism; empowerment; democracy; market

\footnotetext{
* Directeur de recherche émérite au CNRS, Centre Emile Durkheim, Institut d'études politiques de Bordeaux. Adresse postale : Sciences Po Bordeaux; 11 allée Ausone, 33607 Pessac cedex. Adresse email : j.faget@sciencespobordeaux.fr
}

\section{(c)) EY-NC-ND}

Oñati International Institute for the Sociology of Law

Antigua Universidad s/n - Apdo.28 20560 Oñati - Gipuzkoa - Spain

Tel. (+34) 943783064 


\section{Resumen}

El considerable desarrollo de la mediación en los países occidentales en décadas recientes tiene correlación con las insuficiencias y el anacronismo de las regulaciones institucionales y sociales derribadas por la posmodernidad. Las metamorfosis generadas por su éxito no están exentas de disputas entre juristas debilitados por la crisis de la racionalidad jurídica y que defienden su monopolio, y los nuevos profesionales de la mediación provenientes de las ciencias sociales. Por tanto, se enfrentan dos conceptos de la mediación: una visión idealista del empoderamiento y la emancipación de los individuos y una pragmática y mercantil que tiende a judicializar los modelos alternativos de resolución de conflictos.

\section{Palabras clave}

Mediación; institucionalización; posmodernidad; neoliberalismo; empoderamiento; democracia; mercado 


\section{Table des matières / Table of contents / Í ndice}

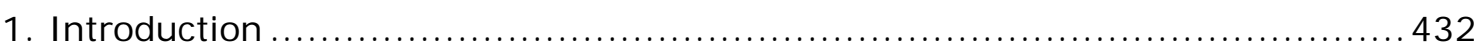

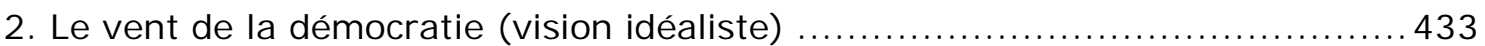

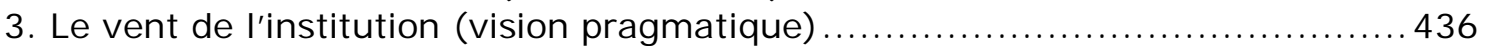

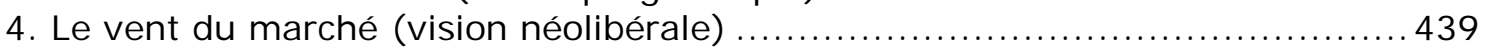

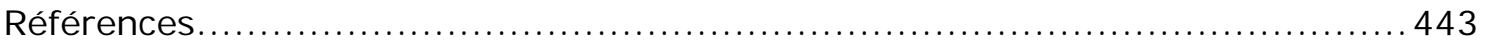




\section{I ntroduction}

Les pratiques de médiation se sont considérablement développées dans les pays occidentaux depuis les années 80. Initiées en Amérique du nord elles se sont d'abord disséminées dans les pays de l'ancien commonwealth partageant des caractéristiques culturelles (protestantisme, conception d'un tat minimal, régime juridique de common law) propices à l'éclosion de modes de régulation contractualisés reposant sur le bargaining. Les programmes de médiation ont ensuite gagné des pays d'Europe continentale parfois catholiques aux systèmes politiques et juridiques différents. Leur essor progressif a engendré une fragmentation spectaculaire des champs d'expériences. Chaque institution a créé ses propres médiateurs, élaboré ses propres formations, ses propres références conceptuelles, sans prendre la mesure de la transversalité de la posture du médiateur. Cette division du travail de médiation n'a pas facilité une claire perception de sa nature. Les représentations sociales de la médiation sont restées et restent encore nébuleuses dans la société et les médiateurs eux-mêmes peinent à situer leur fonction sociale et donc leur identité dans un contexte politique et professionnel global. Leur vision se limitant le plus souvent à leur périmètre d'activité (familial, pénal, scolaire, entreprise, social, sanitaire, interculturel...) ils sont plus ou moins aveugles à tout ce qui le déborde. On peut donc considérer qu'il existe un impensé de la médiation, en particulier par rapport à ses conditions de production et de mise en œuvre (Faget 2008). L'objectif de cet article tentera d'y remédier en développant une vision politique la plus ample possible des différentes expressions de la médiation ainsi qu'une analyse transversale de ses pratiques.

Le développement constant et concomitant de la médiation dans les champs les plus divers n'est pas le fruit du hasard. II s'inscrit dans le contexte d'une métamorphose profonde des modes de pilotage sociaux. II signe le passage de la modernité à la post-modernité (autrement nommée modernité avancée, aiguë, tardive, réflexive, surmodernité, hypermodernité... [Bonny 2004]), peut être illustré par quelques indicateurs aux causalités multiples et enchevêtrées. Le «désenchantement du monde » (Gauchet 1985) du fait de l'effondrement des grands récits religieux et idéologiques, la transformation des institutions publiques et la diminution de la place de l'État dans le jeu social, l'avènement du néo-libéralisme et la stagnation corrélative des ressources de l'État Providence, l'osmose croissante entre secteur public et privé, l'internationalisation de la finance et du capital, la dérégulation des marchés et les logiques de globalisation économique, les phénomènes migratoires, le développement des nouvelles technologies scientifiques et de communication qui bouleversent le rapport au temps et dématérialisent les échanges financiers et les relations humaines, représentent les signaux de l'affaissement de la séquence historique dite moderne et de l'anachronisme des institutions qui l'ont étayée.

Toutes ces complexités font que la société ne peut désormais plus être pilotée par des structures statiques, que sont les lois et les institutions, qui assuraient traditionnellement sa stabilité. II faut désormais rechercher de nouvelles formes flexibles, fluides, adaptables, souples de régulation, susceptibles de rendre un peu de substance à un univers possiblement chaotique. La médiation constitue une de ces formes d'ajustement qui participe, en complément des régulations institutionnelles territorialisées dont les fonctions symboliques et instrumentales sont encore pertinentes, à une articulation d'univers de sens hétérogènes et multiples. J 'ai utilisé la métaphore du fluide (Faget 2005), à l'instar de celle d'une " modernité liquide »(Bauman 2000), pour décrire un contexte historique orphelin de certitudes et de formes sociales solides. Elle vise à montrer que notre capacité à « faire société » se «travaille » désormais dans la création de nouveaux espaces intermédiaires où se bricolent de micro-ajustements. Le fluide est ce qui permet de dénouer au lieu de trancher, de lier au lieu de séparer, de mettre en synergie au lieu d'opposer. Mais dans toutes les mythologies, depuis les contes d'enfant jusqu'aux romans de sciencefiction, c'est aussi une sorte d'énergie occulte, une influence inexplicable émanant de personnages charismatiques ou d'entités mystérieuses. Dans cette acception la 
fluidité de la médiation ne désigne pas seulement ses vertus instrumentales d'écoulement et de régulation mais la dimension magique d'une recherche de sens.

En ce sens les médiations ne constituent pas seulement de simples outils de gestion de la conflictualité sociale. Elles illustrent aussi à leur façon les bouleversements idéologiques affectant nos sociétés. Mais il reste difficile d'en déterminer la substance. Ont-elles une fonction homéostatique ou mutagène ? Autrement dit, assurent-elles la reproduction de logiques institutionnelles préexistantes sous des visages plus avenants ou sont-elles de nature à les transformer en profondeur ? II n'y a pas de réponse globale à cette question. Si les principes déontologiques de la médiation sont relativement cohérents, par contre les différentes déclinaisons institutionnelles des pratiques montrent la confrontation de rationalités diverses et l'interaction d'acteurs multiples dont les objectifs sont souvent ambivalents. Ce sont des objets idéologiques non identifiés dont les enjeux diffèrent en fonction des contextes dans lesquels ils opèrent (Faget 2012). Si les médiations sociales, interculturelles, scolaires, familiales peuvent être analysées comme des fers de lance d'un projet libertaire d'émancipation des individus et des groupes, les médiations civiles et commerciales s'inspirent plutôt d'un projet de dérégulation institutionnelle et normative. En conséquence on ne peut pas utiliser la même grille de lecture pour analyser tous les types de médiations même si les fondamentaux déontologiques (indépendance, impartialité, neutralité, absence de pouvoir de décision, confidentialité...) sont communs à toutes les médiations et leur assure un minimum de cohérence. L'affirmation de la diversité de leurs expressions et de leur sens ne doit cependant pas oublier qu'elles sont actuellement traversées par les mêmes forces, ballottées par les mêmes vents contraires, ceux qui soufflent dans le sens de la démocratie et qui se heurtent aux courants d'air endurants de l'institution et aux rafales du marché.

\section{Le vent de la démocratie (vision idéaliste)}

Il est nécessaire de rappeler que la médiation n'a pas été conçue par quelques gestionnaires du social, mais qu'elle fût portée sur les fonts baptismaux par des mouvements protestants, Mennonites et Quakers, inspirés par la recherche d'un monde meilleur, des militants de la non-violence (inspirés par Gandhi, Martin Luther King puis plus récemment Nelson Mandela) voulant propager un message de tolérance et de compréhension et des juristes critiques « de gauche » dévoilant la nature idéologique des processus de domination que constituent le droit et le système judiciaire. Les critical legal studies (Unger 1983) considèrent que les textes juridiques sont présentés par le libéralisme comme universels mais c'est un tour de passe-passe car en réalité ils constituent une idéologie politique défendant les intérêts des élites politiques et économiques et non pas les valeurs de l'ensemble des membres de la société. La norme juridique est abstraite. Elle ne tient pas compte des besoins particuliers et fait entrer les litiges dans des catégories juridiques préalablement établies et stéréotypées. Elle détruit les relations interpersonnelles au lieu de les construire. La procédure contradictoire ne fait qu'aggraver les conflits, le fait de devoir désigner un gagnant et un perdant rend impossible la recherche d'une entente. La justice formelle fournit des jugements rétrospectifs sans se soucier de réparer ou de reconstruire pour l'avenir ce qui a été brisé.

Cette dénonciation, comme le pacifisme de ses pionniers, révèle que la médiation est initialement pensée comme une contre-culture face aux pouvoirs institutionnels coercitifs dont les modalités d'action sont déshumanisées. Le rappel de ces origines montre que l'essor des pratiques de médiation n'est pas seulement un choix contraint pour mettre de l'huile dans des rouages institutionnels rouillés. Elle comporte aussi un projet de transformation sociale et politique dans sa façon d'ouvrir la voie à une démocratie participative expérimentale, à une politique de reconnaissance et à la promotion d'un individualisme relationnel. 
Ces valeurs humanistes qui considèrent l'être humain comme valeur suprême devant être protégé contre toutes les formes d'assujettissement politique, religieux ou économique, se rencontrent dans les principes éthiques qui irriguent tous les textes fondateurs, qu'ils soient nationaux ou internationaux.

- Une vision positive des potentialités humaines: la médiation glorifie la capacité des individus à exercer des choix en postulant leur compétence dans la régulation de leurs propres conflits. Ce postulat de leur compétence est au cœur d'un processus qui considère, de façon délibérément optimiste, que tout être humain peut à tout moment progresser et changer, améliorer ses capacités d'écoute, de communication et de compréhension.

- Une éthique de la responsabilité : l'individu porte une parole qui lui est propre, s'engage personnellement sans être représenté. Cet objectif s'intègre dans le projet individualiste post-moderne. II marque le glissement d'un imaginaire juridique adossé sur des valeurs collectives à un imaginaire démocratique qui donne à chacun la possibilité d'être producteur de droit. L'éthique de la médiation propose en effet que les conflits ne soient pas réglés par rapport à une norme transcendante (prêt à porter juridique) mais par un accord conclu entre leurs protagonistes (sur mesure juridique). Considérer que chacun est en mesure de devenir son propre législateur représente naturellement une subjectivisation de la norme et constitue une entorse au modèle Jupitérien d'une loi toute-puissante fondée sur la Raison (Ost 1991).

- Une éthique de la fraternité et de l'harmonie sociale: les logiques de coopération, qui nécessitent la découverte de l'altérité, de la tolérance et du respect, doivent supplanter les logiques de la compétition qui président au jeu judiciaire. Ce travail qui permet de passer d'une logique perdant/perdant hystérisant les conflits à une logique gagnant/gagnant, permettant aux protagonistes de découvrir leurs intérêts communs, contribue à la dissémination d'une culture de paix La médiation, grâce à "la parité de participation" (Fraser 2005) qu'elle instaure, ouvre justement un espace d'écoute et de travail sur l'altérité au cours duquel on accède à la connaissance de soi par le détour de la prise de conscience de la singularité de l'autre. Car la compréhension de soi « s'acquiert par le biais de l'intersubjectivité » (Honneth 2000). En substituant à la logique du côte-à-côte celle du face-àface qui permet la confrontation du "je" souverain avec l'autre, qui lui est différent, qui lui résiste et qui en définitive détermine son existence, la médiation permet d'articuler nos identités individuelles et collectives.

- Une éthique d'égalité : la médiation cultive la croyance que les individus, indépendamment de leurs caractéristiques sociales, culturelles et psychologiques, peuvent communiquer entre eux à la condition qu'on leur garantisse une possibilité formelle de le faire. La conception de relations horizontales, ou chacun est considéré comme l'égal de l'autre, relève d'une vision républicaine universaliste ambitieuse dont on peut cependant redouter qu'elle n'occulte l'existence des rapports politiques de domination. L'éthique de médiation doit être pensée dans le cadre général d'une "politique de reconnaissance" (Taylor 1994). Dans un contexte "post-socialiste" ou "l'injustice fondamentale n'est plus l'exploitation mais la domination culturelle" (Fraser 2005), ce besoin de reconnaissance s'exprime par la multiplication de revendications collectives placées sous la bannière de la race, de l'ethnicité, du genre et de la sexualité mais se décline également à l'échelle individuelle par le besoin multiforme d'être entendu, écouté, respecté car "la reconnaissance n'est pas seulement une politesse qu'on fait aux gens: c'est un besoin vital" (Taylor 1994).

- Un projet politique d'émancipation : le travail de médiation a pour objectif de renforcer l'empowerment des individus et des groupes (se rendre maître de son destin, favoriser l'aptitude à défendre ses droits, se libérer de ses chaînes, s'émanciper des situations d'aliénation). Le fait de placer l'individu au coeur 
de la régulation sociale, de le considérer comme porteur de sa propre parole, s'intègre dans le projet individualiste post-moderne. Le glissement d'un imaginaire juridique adossé sur des valeurs collectives à un imaginaire démocratique qui donne à chacun la possibilité d'être soi n'est pas sans danger. Cet individualisme est généralement associé à la dictature du marché, au libéralisme sauvage, aux inégalités. II est considéré comme un facteur d'égoïsme et d'indifférence. Et il est vrai que l'émergence d'une société d'individus rend aléatoire la production d'un monde commun. La culture du « narcissisme » sécrète une incapacité à communiquer, engendre les phénomènes de rejet de l'autre, s'avère destructrice à bien des égards. Mais en même temps, l'individualisme porte de grandes espérances (De Singly 2005). Il est associé à la progression de la défense des droits de l'homme qui place l'individu sujet de droits au cœur du projet social. Sans la philosophie individualiste les femmes n'auraient pas pu s'émanciper, le droit d'expression des minorités n'existerait pas. Mais cet individualisme créateur n'est pas spontané. II est l'aboutissement d'une conquête. La médiation en constitue un des outils. En responsabilisant les personnes, en leur permettant de découvrir l'altérité par le dialogue et la communication, elle les inscrit dans un lien social. Elle participe d'une rationalité communicationnelle, contribue à la création d'un espace public reposant sur une éthique de la discussion (Habermas 1987). L'individu n'y est plus défini par les institutions ou les autorités mais il se définit lui-même au cours d'un processus d'échange et de reconnaissance interpersonnelle. La médiation peut ainsi permettre d'aider les individus à passer d'un individualisme narcissique à un individualisme relationnel qui permet de vivre les uns avec les autres et non les uns à côté des autres. Mais ce passage ne se fait pas aisément du fait de l'émiettement des identités collectives et de l'absence d'une culture de médiation qui verrait les citoyens recourir spontanément à des régulations compréhensives et pacifiques.

Enfin, les principes éthiques de la médiation portent un projet politique de démocratie participative condamnant tout principe de délégation et exaltant la participation directe de chacun dans la résolution des conflits qui le préoccupent ou qui le blessent. Ce projet politique s'exprime pleinement dans le développement des médiations communautaires, sociales ou citoyennes mobilisant des médiateurs bénévoles pour réguler, en dehors des institutions, les conflits de la vie quotidienne qui détériorent le vivre ensemble. Ce vent démocratique s'inscrit dans le contexte post moderne de sociétés d'individus dans lesquelles l'homo democraticus supporte de plus en plus difficilement toute forme de subordination aux institutions, il « tolère mal les limites et les contraintes que lui imposent les institutions (...) et tend à penser qu'il ne peut être représenté que par lui-même » (Schnapper 2002). Du coup, même si les structures politiques traditionnelles restent des rouages importants, une nouvelle forme d'espace public prend progressivement forme dans laquelle les principes de délégation et de subordination hiérarchique cèdent le pas à des engagements plus directs.

On peut dans cette optique observer de nombreux signes d'apparition de nouvelles arènes de débat public. Ainsi l'élaboration d'une « action publique négociée » (Allain 2005) pour relever les défis posés par les questions relatives à la protection de l'environnement et au développement des sciences et des techniques. Des médiations collectives de plus en plus fréquentes sont en effet mises en œuvre sous des vocables divers (conférences de consensus, forums hybrides [Callon et al. 2001], noyaux de participation populaire...) pour des conflits liés à des problèmes environnementaux, (tracé d'une autoroute, d'une rocade, implantation d'un site de traitement des déchets, d'un parc d'éoliennes, tracé de zones de protection de milieux naturels...), d'aménagement (emplacement d'un centre éducatif renforcé pour les jeunes délinquants, d'un équipement de loisirs...) ou d'usage des espaces publics. On voit là émerger de nouveaux modes de construction des décisions politiques. À des décisions autoritaires d'élus, rationalisées par le savoir des experts, se substituent 
parfois des processus plus activement démocratiques dans lesquels tous les acteurs concernés sont informés ou consultés sur les projets en cours.

Ces forces démocratiques se retrouvent aussi à une autre échelle dans le rêve que cultivent les médiations familiales ou en matière pénale, de rapports sociaux plus amènes, plus harmonieux, moins violents. Elles instillent de la coopération dans des univers basés sur un principe de compétition, de l'humain dans des mécanismes impersonnels, de la démocratie délibérative dans des schémas abstraits de représentation, de la réflexivité dans les routines institutionnelles. Ainsi la médiation familiale est souvent présentée par beaucoup de ses spécialistes comme une technique d'accompagnement des bouleversements du modèle familial. Mais quelques évolutions législatives ou la redéfinition de quelques-unes des innombrables professions juridiques ou familiales créées tout au long du développement de l'État providence auraient suffi à assurer une «police des familles » (Donzelot 1977) performante. L'essor de ce type de médiation dans un paysage institutionnel déjà saturé par de multiples intervenants va bien au-delà, à mon avis, de la mutation des dynamiques familiales contemporaines mais révèle la transformation de modes de régulation de la conflictualité sociale moins étatiques et plus contractualisés.

En ce sens nous assistons à une généralisation des dispositifs qui mobilisent le consentement des acteurs. Ils sont liés à la crise contemporaine des formes traditionnelles d'autorité et des modes de régulation basés sur la contrainte. Dans ce processus de contractualisation la part des liens prescrits par la loi régresse au profit des liens consentis par le contrat où, comme le dit Alain Supiot (2000) « I'hétéronomie recule au profit de l'autonomie ». Et la médiation donne l'occasion de pratiquer cet apprentissage démocratique consistant à formuler une parole acceptable, audible, à savoir écouter celle de l'autre, à développer cette ouverture à autrui sans laquelle aucune vie sociale supportable n'est envisageable.

Ces médiations « démocratiques », quelles qu'en soient les expressions, poursuivent un objectif de transformation politique sociale, sociale et institutionnelle. Elles sont souvent pratiquées par des bénévoles formés ou des professionnels dont les modèles de pratique ne sont pas orientés vers la recherche d'une solution mais d'un dialogue, d'une conversation. Car l'expression de ces forces d'émancipation est facilitée par l'adoption de modèles de pratique non directifs, orientés vers la communication. A l'inverse elle paraît entravée par le choix de modèles directifs focalisés sur la recherche d'une solution (Faget 2015). De ce point de vue le modèle transformatif qui laisse aux personnes le soin d'organiser le processus et de choisir le rythme de travail qui leur convient, est le plus démocratique de tous les modèles disponibles (Folger et al. 2010).

\section{Le vent de l'institution (vision pragmatique)}

A la vision d'une médiation comme outil de transformation du monde s'oppose celle d'un simple processus technique de gestion de la complexité institutionnelle. On peut aisément constater - et ce dans tous les secteurs d'activité - que les programmes de médiation sont de plus en plus institutionnalisés, soit soutenus et financés par les pouvoirs publics, soit intégrés dans des dispositions légales qui en font des passages plus ou moins obligatoires avant tout recours à une juridiction.

Les souffles qui poussent vers l'institutionnalisation sont divers. Ils proviennent des médiateurs eux-mêmes quand ils veulent par un soutien financier pérenniser une activité fragile et peu lucrative ou par une consécration règlementaire ou légale obtenir la reconnaissance de leur activité. Mais les courants les plus puissants procèdent des membres des institutions concernées comme les magistrats et les juristes pour l'institution judiciaire. Leurs arguments sont les suivants : 
- proposer des modes plus rapides et moins onéreux de régulation des conflits aux usagers ou justiciables ;

- donner un égal accès aux programmes de médiation à tous les citoyens et justiciables par un développement territorial homogène des pratiques ;

- en cela favoriser la reconnaissance et le développement de la médiation ;

- s'inspirer de la doxa du new public management et gérer la crise instrumentale que traversent les institutions en délégant ou sous-traitant une part de leurs activités au secteur privé dont l'effet est d'en transformer les méthodes de gestion. Dans cette perspective, les critères de l'efficacité des médiations sont appréciées dans leur capacité à mieux gérer en temps et en argent les flux qui asphyxient les institutions ;

- fonder une orthodoxie (promouvoir des bonnes pratiques) afin d'harmoniser l'offre de médiation faite au public ;

- $\quad$ protéger les citoyens en garantissant leurs droits contre des abus possibles de médiation.

La logique des institutions relève de la thermodynamique. Elles s'ouvrent conjoncturellement mais parcimonieusement à des innovations pour lutter contre l'entropie qui les ronge et redorer leur blason de légitimité. Mais cette ouverture étant considérée comme périlleuse elles s'empressent de contraindre ces alternatives en les institutionnalisant, en les introduisant dans le répertoire de leurs compétences, les asservissant ainsi à leurs logiques internes. Ce processus procède à la manière d'un poumon qui se remplit et se vide, inspire et expire dans un enchaînement mécanique continu, sans espoir de repos. Ce mouvement dialectique entre un ordre vertical qui veut conserver la maîtrise du jeu et un ordre horizontal qui tente d'en modifier les règles, touche toutes les institutions et tous les types de médiation même s'ils ne se déclinent pas tout à fait de la même façon selon le champ d'activité, judiciaire, commercial, environnemental, hospitalier, social, ou politique...

Loin de changer les logiques internes d'un système, les médiations seraient alors une manière d'habiller d'un voile démocratique des mécanismes de pouvoir restés verticaux. Elles relèveraient alors d'une sorte de soft power (Nye 1990) assurant par la dépolitisation et la dérégulation les basses œuvres du néo-libéralisme et du cynisme institutionnel. On doit se rappeler que l'essor des pratiques de médiation est concomitant de l'affaiblissement des États et de l'apparition dans le vocable politique du terme de «gouvernance » pour désigner des modes de gouvernement moins verticaux et autoritaires, plus contractualisés et consensuels, que par le passé.

On l'observe au niveau international dans la régulation des conflits politiques où les Etats délèguent leurs prérogatives à une sorte de « diplomatie parallèle », exercée par des acteurs non étatiques aux statuts divers, (fondations, autorités morales et religieuses, institutions universitaires). Ces initiatives qui représentent le complément fonctionnel masqué d'une diplomatie officielle souvent improductive (Faget 2011) peuvent être analysées comme une stratégie de dépolitisation du travail de pacification (Lefranc 2006).

L'organisation de médiations environnementales dont on a parlé plus avant n'est souvent qu'un faux semblant. Dans la graduation classique des niveaux de participation sociale qui va de l'information à la co-décision en passant par la consultation et la concertation, ces instances se situent le plus souvent à un niveau intermédiaire, car elles dépendent de projets et de propositions préconstruits, et il est rare qu'elles soient investies d'un réel pouvoir de décision qui les constitueraient en authentiques médiations.

Toutes les médiations judiciaires sont confrontées directement à cette collision (Faget 2015) entre logiques contradictoires. Leur effort pour les faire cohabiter dans un respect mutuel est méritoire mais il ne peut être récompensé compte tenu du poids énorme des logiques judiciaires. 
TABLE 1

\begin{tabular}{|l|l|}
\hline \multicolumn{1}{|c|}{ Justice } & \multicolumn{1}{c|}{ Médiation } \\
\hline Logique verticale & Logique horizontale \\
Recherche de la vérité & Recherche de \\
Ordre public & l'équilibre \\
Principe de rupture & Besoins privés \\
Référence au droit & Principe de lien \\
Résolution du litige & Référence à l'équité \\
& Régulation du conflit \\
\hline
\end{tabular}

Table 1 . Logiques judiciaires et logiques de médiation.

La médiation en matière pénale, plus que toute autre, car elle se confronte à un système dont la violence légitime est le mode de fonctionnement, illustre cette relation impossible (Faget 2009a, 2009b). Initiée dans une philosophie humaniste hostile à la binarité réductrice du système répressif, elle a connu rapidement un développement significatif en France mais pour des raisons qui furent davantage rattachées à des impératifs de gestion des flux qu'à des désirs de rupture. Les stratégies développées par les procureurs pour infléchir l'éthique de la médiation et asservir les médiateurs aux logiques judiciaires sont la marque de son instrumentalisation plus que l'amorce d'un glissement du paradigme pénal punitif vers un modèle restauratif (Faget 1997). Car la confrontation des magistrats avec des corps moins professionnalisés, moins structurés et moins prestigieux, relève le plus souvent du rapport de domination et toutes les pratiques sociales institutionnalisées par l'appareil judiciaire sont peu ou prou largement instrumentalisées et courent le risque de perdre leur âme (Faget 2015). ${ }^{1}$

Pour ces institutions les médiations sont considérées surtout, le diagnostic peut varier en fonction du contentieux, comme des techniques de résolution des conflits et de facilitation des relations contractuelles. Une bonne médiation est avant tout une médiation rapide qui débouche sur un accord écrit dont les modalités sont consonantes avec les catégories juridiques existantes.

Le développent irrésistible depuis ces dernières années dans tous les pays des médiations judiciaires exprime très clairement cette «captation de la médiation par le droit » (Gaddi 2012) en faisant de la médiation une façon amiable et non plus alternative de règlement des conflits. Organiser l'injonction d'information à la médiation, rendre obligatoire le recours préalable à la médiation avant toute saisine d'un tribunal, l'encadrer juridiquement toujours plus, donner aux juristes la primauté dans sa mise en œuvre et dans son déroulement, c'est courir le risque de transformer

${ }^{1}$ On peut donner de nombreuses illustrations de cette domination de la logique judiciaire:

Les personnes ne peuvent choisir par elles-mêmes la médiation mais sont dépendantes du choix du magistrat ;

Lorsque le magistrat propose la médiation le consentement des personnes n'est pas totalement libre, on dit qu'il est « sous pression » judiciaire ;

Le choix du médiateur ou de son association est fait par un magistrat ;

Le médiateur tire sa rémunération des mesures que lui envoie le magistrat mandant ce qui l'installe dans un rapport de dépendance financière ;

La lecture du dossier pénal par le médiateur se fait au détriment de la dimension humaine du conflit car il transmet une réalité pré construite par l'idéologie professionnelle des policiers ;

Le temps de la médiation s'adapte aux nécessités du temps judiciaire ;

L'exigence implicite de productivité des magistrats conduit les médiateurs à adopter des attitudes plus directives et notamment à proposer des solutions pour augmenter le nombre d'accords ;

Le médiateur a une obligation de rendre compte au magistrat mandant ce qui pose la question de la confidentialité de ce qui se dit en médiation ;

L'homologation terminale de l'accord par un magistrat peut constituer une entrave au caractère consensuel de l'accord. 
ce qui n'est qu'un processus en une procédure aux antipodes de l'idéal démocratique qui l'a initiée. Ce qui fait dire à Daniela Gaddi que « chaque médiation réalisée dans le cadre du droit empêche, plutôt qu'elle ne facilite, toute évolution des rapports interpersonnels et des personnes elles-mêmes. Et ce, avant tout, parce que son objectif est le même que celui du droit, à savoir la résolution d'un problème, tandis que (...) la médiation a pour objectif la restauration d'une relation ». Pour elle «le droit interrompt l'échange (...) interdit d'approfondir et, en dernière instance, de penser » (Gaddi 2012). Cette « juridification » progressive de la médiation s'exprime de manière très concrète par l'introduction de la routine professionnelle des juristes dans son déroulement. C'est ainsi qu'apparaissent des dispositions qui lui étaient totalement étrangères jusque alors comme des demandes de provisions au début du processus, la contraction d'assurances professionnelles pour les médiateurs, un calcul des coûts de médiation basé sur le même barème horaire que les prestations juridiques traditionnelles.

Toutefois si la dimension normalisatrice de l'institutionnalisation des médiations est peu discutable, on ne doit pas occulter le fait qu'elles aménagent des espaces de parole inédits au sein de systèmes qui en étaient dépourvus. Ainsi le fort degré d'émancipation des individus proposé par la médiation familiale pourtant de plus en plus encadrée par les normes juridiques n'est pas complètement érodé par le cadre judiciaire. La médiation scolaire surtout quand elle est le vecteur d'une whole school approach transforme l'ensemble des logiques des établissements (elle se situe alors dans une vision idéaliste) tandis que les modèles qui se contentent de réguler les petites bagarres ou insultes de cours de récréation ne représentent qu'un gadget pédagogique insusceptible d'initier de nouvelles utopies éducatives. Les médiations communautaires pratiquées par des médiateurs bénévoles et dont la fonction est de gérer les petits conflits de la vie quotidienne, les médiations interculturelles qui, à la frontière du travail social, ont pour mission de favoriser le dialogue entre des populations aux racines culturelles diverses, peuvent échapper à l'emprise des financements qu'elles reçoivent à la condition de savoir résister à leur instrumentalisation politicienne (Faget 2015). Mais la voie pour des pratiques indépendantes des institutions et des logiques marchandes et concurrentielles.

\section{Le vent du marché (vision néolibérale)}

L'arrivée des médiateurs dans le champ de la régulation des conflits a provoqué la méfiance voire l'ire des juristes, surtout les avocats, qui en détiennent historiquement le monopole. Ils n'ont pas su, dans leur grande majorité, comprendre et donc anticiper la crise de la rationalité juridique et ont vu débarquer dans leur pré carré des praticiens de la relation qu'ils considèrent comme leurs ennemis. Face à eux ils ont déployé d'ardentes stratégies de défense pour protéger leur monopole et leurs territoires professionnels écornés. Et de fait leurs puissants lobbies ont su manœuvrer à la fois sur les autorités politiques et judiciaires pour influer sur le contenu des textes législatifs et règlementaires. Le dernier exemple en date est fourni par une note du ministère français de la Justice du 8 février $2018^{2}$ précisant que « l'exercice de l'activité de médiation n'est pas subordonné à la détention d'un quelconque diplôme. Ainsi, le diplôme d'État de médiateur familial (DEMF) (...) ne constitue pas un préalable à la pratique de la médiation familiale ». Avec ce texte toutes les professions juridiques, avocats, notaires et huissiers, pourront donc pratiquer la médiation, y compris la médiation familiale dont les spécificités psychologiques exigent pourtant un solide bagage, sans devoir justifier d'une formation en matière de médiation. Cette note rédigée pour organiser la médiation judiciaire, sera, à n'en pas douter, mise à profit par les juristes pour revendiquer une compétence générale pour toutes les médiations conventionnelles. Une telle ouverture du marché à la libre concurrence laissera peu d'espace aux médiateurs non juristes qui seront

\footnotetext{
${ }^{2}$ Dépêche du 8 février 2018 présentant certaines dispositions du décret n²017-1457 du 9 octobre 2017 relatif à la liste des médiateurs auprès de la cour d'appel.
} 
probablement laminés par la puissance du lobby des professions juridiques. Ne seront épargnés que les médiateurs « sociaux » peu concurrentiels qui interviennent dans des champs d'activité peu ou pas lucratifs. Ce choix ministériel prend à contre-pied les observations faites par les spécialistes de la médiation qui estiment qu'il n'est en aucune façon nécessaire de posséder une formation juridique pour pratiquer la médiation. Car à quoi bon mobiliser des compétences juridiques dès lors qu'on ne travaille pas sur le litige, les raisons et les torts, mais sur le conflit dont la nature est le plus souvent obscure, insaisissable, irrationnelle. A quoi bon être juriste alors que le médiateur n'a pas à dire la loi, à donner des conseils juridiques, à juger de la pertinence juridique de la solution envisagée par les protagonistes. Ce sont ces derniers, éventuellement conseillés par leurs défenseurs, qui ont ce rôle. Et le juge garde toujours la possibilité de ne pas homologuer, in fine, un accord qui ne lui paraîtra pas conforme à l'intérêt mutuel des personnes ou contreviendrait à des dispositions d'ordre public. Indépendamment de son background initial, le cœur de la compétence d'un médiateur réside essentiellement dans l'acquisition et la mise en œuvre de techniques de communication. Une conscience juridique lui sera toujours utile, mais une culture juridique plus rarement et uniquement dans certains types de contentieux très techniques (Faget 2015).

Cette évolution est paradoxale si l'on se souvient que les premières médiations ont été conçues dans l'objectif de désinstitutionnaliser et de déprofessionnaliser la gestion des conflits interpersonnels et sociaux. Or c'est tout l'inverse qui se produit ici. Les intérêts corporatistes ont pris le dessus sur les défenseurs d'un intérêt général recherchant à fonder un nouveau contrat social, une manière plus pacifiée de vivre ensemble. On pouvait se douter d'une telle évolution au regard du succès rencontré aux États-Unis dès la fin des années 1970 par les ADR (Alternative dispute resolution) dans le domaine commercial. Le poids de la doxa néolibérale dans les années 1980 et 90 en ont accentué les effets. La réduction croissante du rôle de l'État entraîna corrélativement la crise de la rationalité juridique qui fut un des piliers de la construction des États modernes.

Les modes autrefois dénommés alternatifs de régulation des conflits sont devenus par la magie du législateur français, mais aussi des textes européens, de gentils processus amiables. Ce changement sémantique est emblématique d'un changement de sens. Au lieu de proposer une autre voie, à l'écart de l'institution judiciaire et de ses courtisans, le terme amiable indique clairement que l'on reste dans le même champ institutionnel et donc dans la même logique. On substitue simplement, mais ce n'est toutefois pas un mince progrès, un esprit de négociation aux anciennes joutes mortifères. Et l'on en réserve la mise en œuvre aux mêmes acteurs qui autrefois animaient les combats judiciaires et qui continueront à le faire puisqu'ils auront une double compétence de défenseurs et de médiateurs. Ce passage complexe d'une posture à l'autre ne tardera probablement pas à provoquer des crises identitaires dans un personnel judiciaire désormais écartelé entre deux registres antinomiques.

Ce double registre de compétence a pour effet de transformer l'organisation du travail juridique, entre une activité qui ne l'est pas (juridique) et une autre qui le reste, mais aussi de bousculer la représentation sociale des professions concernées qui éprouveront des difficultés à articuler une image de stricte impartialité avec celle d'une protection sans faille, la vision d'un pacificateur avec celle d'un combattant. La structuration des entreprises juridiques en est modifiée puisque la médiation exige l'ouverture de lieux neutres et provoque progressivement une répartition des tâches au sein des cabinets sur le modèle bien connu des law firms américaines, ces grandes usines juridiques couvrant l'éventail de toutes les prestations juridiques (procès, contrats, bargaining, négociation, médiation...) et constituées de spécialistes dans chacune d'elles. Ces «marchands de droit » (Dezalay 1992) n'hésitent pas à développer des stratégies de marketing agressives de conquête du marché. Et la médiation n'échappe pas à cette compétition. 
Dans cette optique mercantile les médiations sont considérées comme une technique de résolution des conflits et de facilitation des relations contractuelles. Les praticiens qui sont dans ce cas adoptent des modèles de communication relativement structurants et fermés. Leur posture est relativement directive, ils organisent le processus, focalisent le débat sur les éléments juridiquement, économiquement ou stratégiquement pertinents. Ce modèle n'a pas pour objectif de transformer les individus, leur rapport au monde, mais seulement le contexte conflictuel qui les oppose. Certes les acteurs y jouent un rôle privilégié que les usages institutionnels antécédents leur dérobaient, ils ont une part essentielle dans la recherche des solutions, mais le médiateur reste le maître du jeu. C'est un processus d'autant plus subtil de normalisation des comportements que ses participants y consentent. L'écrasante domination du modèle de la négociation raisonnée (Fisher et Ury 1982) dans les médiations civiles, commerciales ou d'entreprise marque la recherche d'un ordre négocié en lieu et place d'un ordre juridique imposé considéré comme peu performant et de moins en moins légitime. L'explosion des modes alternatifs (ou amiables) de résolution des conflits, serait, comme je l'ai déjà évoqué, l'expression d'une contractualisation de la société (Supiot 2000), face à la crise instrumentale et de légitimité du système judiciaire. On a pu dénoncer les dangers d'un tel modèle libéral dont la tendance serait de noyer les inégalités sociales dans des processus de délibération pour lesquels les groupes dominants sont mieux préparés (Fraser 2005). La désertion des formes rituelles publiques de la régulation des conflits au profit de leur privatisation procédurale croissante entraverait la prise en compte de leur dimension politique et serait, in fine, une manière d'en occulter l'existence.

Ce ne sont pas seulement les pratiques de médiation qui se sont marchandisées mais aussi la formation à la médiation. Son importance croissante en France mais aussi en Europe (j'ai eu l'occasion de participer à de nombreux programmes de formation en Suisse, en Belgique, en Italie, en Espagne) est quelque peu déconnectée des besoins sociaux car le discours de la médiation est bien plus développé que sa pratique, les offres de formation trop nombreuses par rapport aux débouchés professionnels qu'elles offrent. Du coup beaucoup de diplômés en médiation n'exploitent pas des compétences acquises et pour le moins inégales. Car à côté de quelques cursus sélectifs (réalisation de mémoires, stage obligatoire, rapport de stage, mises en situation, épreuves pratiques finales) et exigeants (nombre d'heures de formation élevé, déconstruction des savoirs précédemment acquis, travail sur soi approfondi, connaissance de tous les modèles de médiation et non pas du seul modèle dit d'Harvard de la négociation raisonnée...) se répandent des offres de formation (parfois par internet) axées sur l'apprentissage rapide de techniques sommaires et dont le mode de sélection est essentiellement basé sur le coût exorbitant des frais d'inscription. On peut alors sérieusement redouter que le recrutement des médiateurs se fasse davantage par l'argent que par le mérite...

Entre ces trois forces venteuses de la démocratie, de l'institution et du marché, existent naturellement des échanges et des superpositions. On peut être à la fois idéaliste, soucieux du droit des usagers et préoccupé par sa propre survie économique. Mais malgré ces phénomènes d'osmose, mes observations en France et ailleurs me conduisent à penser que les forces de l'institution et du marché prennent progressivement le pas sur celles de l'inspiration pour les soumettre à une rationalité juridique dont ces dernières s'étaient données initialement pour mission de réduire la portée.

De fait, un fossé se creuse de plus en plus profondément entre deux conceptions de la médiation. Une summa divisio oppose des modèles axés sur la recherche rapide d'une solution (vision néolibérale d'une société pragmatique, efficace, technique qui « gère » les litiges dans une perspective de court terme), la production de cessez-lefeu ou pour reprendre l'expression de Johan Galtung, d'une paix négative, et ceux axés sur la lente construction d'une communication, d'une conversation (vision d'une 
société plus réflexive, plus soutenable, qui « régule » les conflits dans une optique de long terme), de la construction d'une paix positive.

Cette dualité de conceptions s'exprime dans la fonction politique accordée aux médiations. Pour les uns les modes « amiables » pactisent avec les principes et les routines de l'ordre institutionnel et contribuent à son homéostasie, pour les autres les modes «alternatifs» ont pour ambition d'en subvertir les logiques. Les médiations judiciaires sont à la croisée de ces deux intentions, si bien qu'elles constituent une créature institutionnelle hybride dont il est bien difficile de définir la vraie nature, écartelées entre le désir de liberté, la soif d'émancipation qui les fondent et leur mutation empirique en une contrainte acceptée.

Ces trois dynamiques de la médiation peuvent se synthétiser en réalité en deux approches pratiques, deux façons de concevoir et de faire la médiation, les forces institutionnelles et néolibérales, quoique distinctes, partageant une même vision pragmatique.

\section{TABLE 2}

\begin{tabular}{|l|l|}
\hline \multicolumn{1}{|c|}{ Vision Idéaliste } & \multicolumn{1}{c|}{ Vision Pragmatique } \\
\hline Travail sur le conflit. & Travail sur le litige. \\
$\begin{array}{l}\text { Le conflit peut avoir des fonctions } \\
\text { positives. }\end{array}$ & Le conflit est un problème. \\
Recherche d'une communication. & Recherche d'une solution. \\
$\begin{array}{l}\text { Posture du médiateur plutôt non } \\
\text { directive. }\end{array}$ & Posture du médiateur plutôt directive. \\
Compétences psycho sociales. & Compétence juridique éventuelle. \\
Accent porté sur le temps psychique & Accent porté sur la vitesse du \\
nécessaire (médiations plus longues). & processus (médiations plus brèves). \\
Accueil des émotions. & Evitement des émotions. \\
Travail sur l'altérité. & Travail sur le rapprochement des \\
Gratuité ou coût réduit. & positions. \\
Encouragement à la créativité dans la & Coût basé sur celui des prestations \\
recherche de solutions. & juridiques. \\
\hline
\end{tabular}

Table 2. Deux visions de la médiation.

Le temps n'est plus de se demander si la médiation est un phénomène de mode. La crise inexorable de la rationalité juridique dans nos sociétés complexes ouvre des temps nouveaux. Alors que la réponse judiciaire et institutionnelle était depuis le début du XIXème siècle le principe, désormais les modes alternatifs de règlement des conflits deviendront l'exception et l'État n'interviendra qu'en vertu d'un principe de subsidiarité. Une nouvelle forme de contrôle social se met donc en œuvre, dont la médiation donne une belle illustration, qui repose non plus sur la contrainte mais sur le consentement et la participation des individus. Apparemment cela va dans le sens d'une démocratisation de la démocratie. Pourtant il est permis de se demander si cette évolution, à la croisée de chemins idéalistes, gestionnaires et marchands, sera le ferment d'une émancipation des individus ou l'expression de leur servitude volontaire. 


\section{Références}

Allain, S., 2005. La médiation environnementale comme système de régulation politique. Application au gouvernement de l'eau. Dans : J. Faget, dir., Médiation et action publique : la dynamique du fluide. Pessac: Presses Universitaires de Bordeaux, pp. 135-150.

Bauman, Z., 2000. Liquid Modernity, Cambridge: Polity Press.

Bonny, Y., 2004. Sociologie du temps présent: Modernité avancée ou postmodernité ? Paris : Armand Colin.

Callon, M., Lascoumes, P., et Barthe, Y., 2001. Agir dans un monde incertain : Essai sur la démocratie technique. Paris : Seuil.

De Singly, F., 2005. L'individualisme est un humanisme. Paris : L'Aube.

Dezalay, Y., 1992. Marchands de droit : La restructuration de l'ordre juridique international par les multinationales du droit. Paris : Fayard.

Donzelot, J., 1977. La police des familles. Paris : Minuit.

Faget, J., 1997. La médiation : essai de politique pénale. Toulouse : Erès.

Faget, J., 2008. L'impensé de la médiation. Contre culture ou soft power ? Empan, 2008/4(72), pp. 74-80.

Faget, J., 2009a. I "raggionevoli compromessi" della mediazione penale. Studi sulla questione criminale, 4(1), pp. 59-68.

Faget, J., 2009b. Les accommodements raisonnables de la médiation pénale, Revue de science criminelle et de droit pénal comparé, $n^{\circ} 4$, octobre-décembre.

Faget, J., 2011. The metamorphosis of peacemaking. Dans: J. Faget, ed., Mediation in Political Conflicts: Soft Power or Counter Culture ? Oxford: Hart, pp. 1-28.

Faget, J., 2012, As vidas divididas da mediaçao. Meritum [en ligne], 7(2julho/dizembro), pp. 229-247. Disponible sur: http://www.fumec.br/revistas/meritum/article/view/1602/1003 [Consulté le 18 janvier 2019].

Faget, J., 2015. Médiations, les ateliers silencieux de la démocratie. 2ème ed. Toulouse: Erès.

Faget, J., ed., 2005. Médiation et action publique : La dynamique du fluide. Pessac: Presses Universitaires de Bordeaux.

Fisher, R., et Ury, W., 1982. Comment réussir une négociation? Trad.: L. Brahem. Paris: Seuil.

Folger, J.P., Bush, R.A.B., and Della Noce, D.J ., eds., 2010. Transformative Mediation: A Source Book, Resources for Conflict Intervention Practitioners and Programs. Dayton, $\mathrm{OH}$ : Institute for the study of conflict transformation.

Fraser, N., 2005. Qu'est-ce que la justice sociale ? Reconnaissance et redistribution. Trad.: E. Ferrarese. Paris : La Découverte.

Gaddi, D., 2012. Les effets indésirables du processus d'assimilation de la médiation par le droit. Informations sociales, $n^{\circ} 170$, pp. 28-34.

Gauchet, M., 1985. Le désenchantement du monde : une histoire politique de la religion. Paris : Gallimard.

Habermas, J., 1987. Théorie de l'agir communicationnel. Paris : Bayard.

Honneth, A., 2000. La lutte pour la reconnaissance. Trad. : P. Rusch. Paris : Cerf.

Lefranc, S., 2006. Le mouvement pour la justice restauratrice. "An idea whose time has come". Droit et société [en ligne], 63/64(2), pp. 393-409. Disponible sur: 
https://www.cairn.info/revue-droit-et-societe1-2006-2-page-393.htm [Consulté le 18 janvier 2019].

Nye, J.S., 1990. Bound to Lead: The Changing Nature of American Power. New York: Basic books.

Ost, F., 1991. Jupiter, Hercule ou Hermès, trois modèles du juge. Dans : P. Bouretz, dir., La force du droit. Paris : Esprit, pp. 241-272.

Schnapper, D., 2002. La démocratie providentielle : essai sur l'égalité contemporaine. Paris: Gallimard.

Supiot, A., 2000. La contractualisation de la société. Dans : Y. Michaud, dir., Qu'est-ce que I'humain ? Paris: Odile Jacob.

Taylor, C., 1994. Multiculturalisme : Différence et démocratie. Paris : Flammarion.

Unger, R.M., 1983. The Critical Legal Studies Movement. Cambridge, MA: Harvard University Press. 